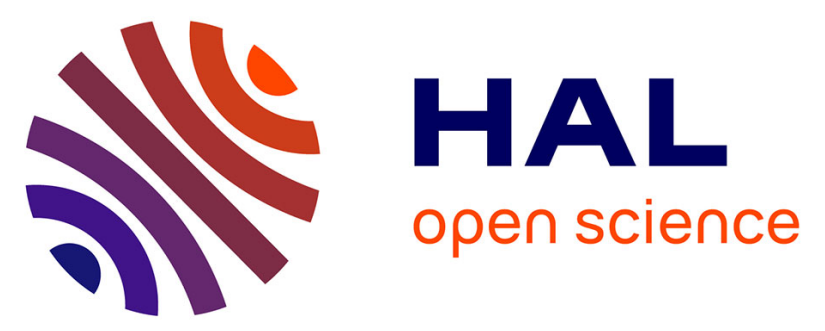

\title{
Interactive Genetic Algorithm to Collect User Perceptions. Application to the Design of Stemmed Glasses
}

\author{
Jean-François Petiot, David Blumenthal, Emilie Poirson
}

\section{To cite this version:}

Jean-François Petiot, David Blumenthal, Emilie Poirson. Interactive Genetic Algorithm to Collect User Perceptions. Application to the Design of Stemmed Glasses. Fouad Bennis; Rajib Kumar Bhattacharjya. Nature-Inspired Methods for Metaheuristics Optimization, 16, Springer, pp.35-51, 2020, Modeling and Optimization in Science and Technologies, 10.1007/978-3-030-26458-1_3 . hal02493595

\section{HAL Id: hal-02493595 \\ https://hal.science/hal-02493595}

Submitted on 12 Apr 2021

HAL is a multi-disciplinary open access archive for the deposit and dissemination of scientific research documents, whether they are published or not. The documents may come from teaching and research institutions in France or abroad, or from public or private research centers.
L'archive ouverte pluridisciplinaire HAL, est destinée au dépôt et à la diffusion de documents scientifiques de niveau recherche, publiés ou non, émanant des établissements d'enseignement et de recherche français ou étrangers, des laboratoires publics ou privés. 


\title{
Interactive Genetic Algorithm to Collect User Perceptions. Application to the Design of Stemmed Glasses
}

\author{
E. Poirson, J.-F. Petiot, and D. Blumenthal
}

\begin{abstract}
To avoid flops, the control of the risks in product innovation and the reduction of the innovation cycles require valid and fast customer's assessments. A methodology must be proposed to the designer to take into account the perceptions of the user. The method presented is based on an iterative process of user selection of representative CAD models of the product. An IGA is used to interpret the user's choices and introduce new products. In the center of this methodology, the user who, thanks to his decisions, will guide the evolution of the algorithm and its convergence. After a description of the IGA, a study on the convergence of the IGA is presented, according to the tuning parameters of the algorithm and the size of the design problem. An experiment was carried out with a set of 20 users on the application case proposed a steemed glass. The implementation of the perceptive tests and the analysis of the results, using Hierarchical Ascendant Classification (HAC) is described. The main contributions of the paper are proposals of (1) an interactive product optimization methodology; (2) a procedure for parameterizing interactive genetic algorithms; (3) a detection of perceptive trends that characterize customer expectations; (4) an experimental application on a real life product.
\end{abstract}

Keywords Interactive genetic algorithms - Shape design - Convergence · Perceptive tests · Design methodology · Genetic operators

E. Poirson $(\bowtie) \cdot$ J.-F. Petiot

LS2N, Ecole Centrale de Nantes, Nantes, France

e-mail: Emilie.poirson@ec-nantes.fr; jean-francois.petiot@ec-nantes.fr

D. Blumenthal

AgroParistech, Massy, France

e-mail: david.blumenthal@agroparistech.fr 


\subsection{Introduction}

To allow the companies to remain competitive, the current society imposes times of development of product/service shorter and shorter, products cheaper and cheaper and always better quality. The leitmotif of the Quality Function Deployment methodology is for illustration "the right product the first time and each time". QFD is a method of introducing quality at the design stage to satisfy the customer. The method helps product designers to explicitly identify the needs of consumers, correlate them with the technical characteristics given by the engineers, and evaluate the potential characteristics of the product compared to those already existing in the market [1]. The QFD is adapted for performance indications and product usages study. For example, [25] presents a methodology to mathematically calculate the relation between the technical customer requirements and the technical product characteristics. This methodology is used to fill the house of quality tool of the quality function deployment method during the product design and development.

This methodology is part of the user-oriented design research topic. One of the major points of this orientation is taking into account not only the expected performances but also the perceptions or emotions that the client wishes to experience. To understand and integrate them throughout the product development cycle, verbalization is regularly used. The language causes problems whether in differences of translation or definitions of the verbatim between qualifications of participants [18]. Indeed, on hedonic studies for which a population of naive subjects is questioned, the precision in the vocabulary misses accuracy compared to the expert subjects who used a combination of both descriptions and hedonic terms when describing a product (wine in [11]), indicating that they are better at communicating and describing what they like. It is particularly difficult to verbalize certain perceptions, and even more emotions. To overcome this difficulty, pictorial representations are used, as PrEmo [2], tools that uses illustrated characters represented the 5 basics emotions. These methods are part of the current Kansei Engineering, a method founded by Mr. Nagamachi at Hiroshima University about 30 years ago. Kansei engineering is used to quantify people's perceptions and to translate them into the design elements. Sensory tests, statistical methods, and AI techniques have been applied to formalize these relationships. Various modeling methods are proposed to provide useful design rules or trend prediction [17, 29].

Another branch of the literature of user oriented design is human-computer interactions where an algorithm gradually refines the propositions made to the users, based on their previous assessments. An example of this is interactive evolutionary computation (IEC), where the user is used as an evaluator in an evolutionary process [27]. In classical EC, a mathematical function evaluates the fitness (adaptation of the population to the environment). In IEC, this function is implicit and is provided by the assessments of the user. Since the user evaluates the fitness, there is no need for a prior mathematical function. The intervention of humans to replace the 
mathematical evaluation of fitness has been applied in many fields (music, writing, education, food industry, etc.) involving different sensory modalities. This IEC method has also been used in a number of fashion applications [15].

Let's firstly present the family of algorithms chosen for the study: the genetic algorithm.

\subsection{Background on Genetic Algorithms}

\subsubsection{Definition}

Genetic algorithms are evolutionary optimization methods developed originally by Holland [9]. They are based on iterative generations of population of individuals, converging step-by-step toward solutions. The term individual refers here to a specific set of values for the design variables. In other words, an individual is a specific candidate design. A metaheuristic is used to improve the current population of solutions. Based on the principle of Darwins natural evolution theory [3], the algorithm proceeds to a selection of parents, which spread their genetic dominant heritage in the next generation. The general principle of a genetic algorithm can be decomposed in 3 steps. (1) Consider an initial population of individuals randomly created, arbitrarily chosen by the designer, or generated by another calculation process. (2) This population iss evaluated according to the constraints and objectives formulated in the optimization problem. If the stopping criteria of the algorithm are satisfied, the algorithm stops; otherwise (3) the genetic operators are applied to this population in order to change it to a new population of the individuals that best meet the requirements. The steps $2-3$ are repeated till satisfaction of the mathematical fitness.

Genetic algorithms are often used in the literature to explore design space, encourage creativity [23] or to help innovation [19].

\subsubsection{Encoding of the Design Variables}

Each chromosome represents a particular design. For each design, we can define design factor. Each factor can thus take different values called levels. A chromosome containing one level for each variable represents a particular combination, thus design. A binary string represents a variable where the length of the string depends on the number of allowed levels for the variables. For example, a product defined by 5 factors on 4 levels will be encoded by a vector of 5 patterns of 2 bits (4 combinations). 


\subsubsection{The Genetic Operators}

Genetic operators have two objectives: to converge the current population of individuals to a set of optimal solutions (process efficiency) and to explore the largest proportion of the design space (process diversification). These operators are selection, crossover, and mutation. Each operator is performed randomly on individuals.

The selection is a recopy of an individual. In the implementation here, the crossover is a single point crossover: from two individuals, a point of crossing is selected and the two headpieces are swapped. One of the two children is randomly selected. The mutation is a change of one randomly selected factor value. The crossover rate $(\mathrm{Rc})$, mutation $(\mathrm{Rm})$, and selection $(\mathrm{Rs})$ are real values chosen between 0 and 1 such that $\mathrm{Rc}+\mathrm{Rm}+\mathrm{Rs}=1$. Each selected chromosome will therefore undergo one of these 3 operations.

One of the main points of the genetic algorithm is the fitness function that allows the evaluation of the adaptability of the design in the environment. We have already discussed the difficulty of objectifying a function when studying perceptions. One solution is to replace the fitness evaluation by a human one, thus to let the system be interactive.

\subsection{Interactive Genetic Algorithm}

\subsubsection{Synoptic of the IGA Process}

Classically, the fitness evaluation of the individuals concerned is calculated numerically with a mathematical function known beforehand. A particular category of GA, Interactive Genetic Algorithms (IGA), introduces the user in the optimization loop to assess the fitness. During each iteration of the process, the user selects solutions that he/she considers to be the closest to the desired objective. After a number of iterations, the method may converge toward one or several solutions that fulfill the users objective. These algorithms are used to explore design spaces [13]. Since the user decides the individuals fitness, there is no need for a prior and unique formulation of the fitness function. For some applications, such as finding the semantic dimensions of a product, this advantage is crucial.

The general process of the IGA can be seen in Fig. 3.1. The IGA creates an initial population of designs by generating the chromosomes, and presents them to a user as CAD drawings. The number of individuals in a population is chosen by the experimenter according to the number of variables and levels and the size of the screen to represent the designs if visual experiment.

Based on personal criteria the user then selects a subset of these individuals. This number is also a part of the setting up. Selection consists in choosing, 


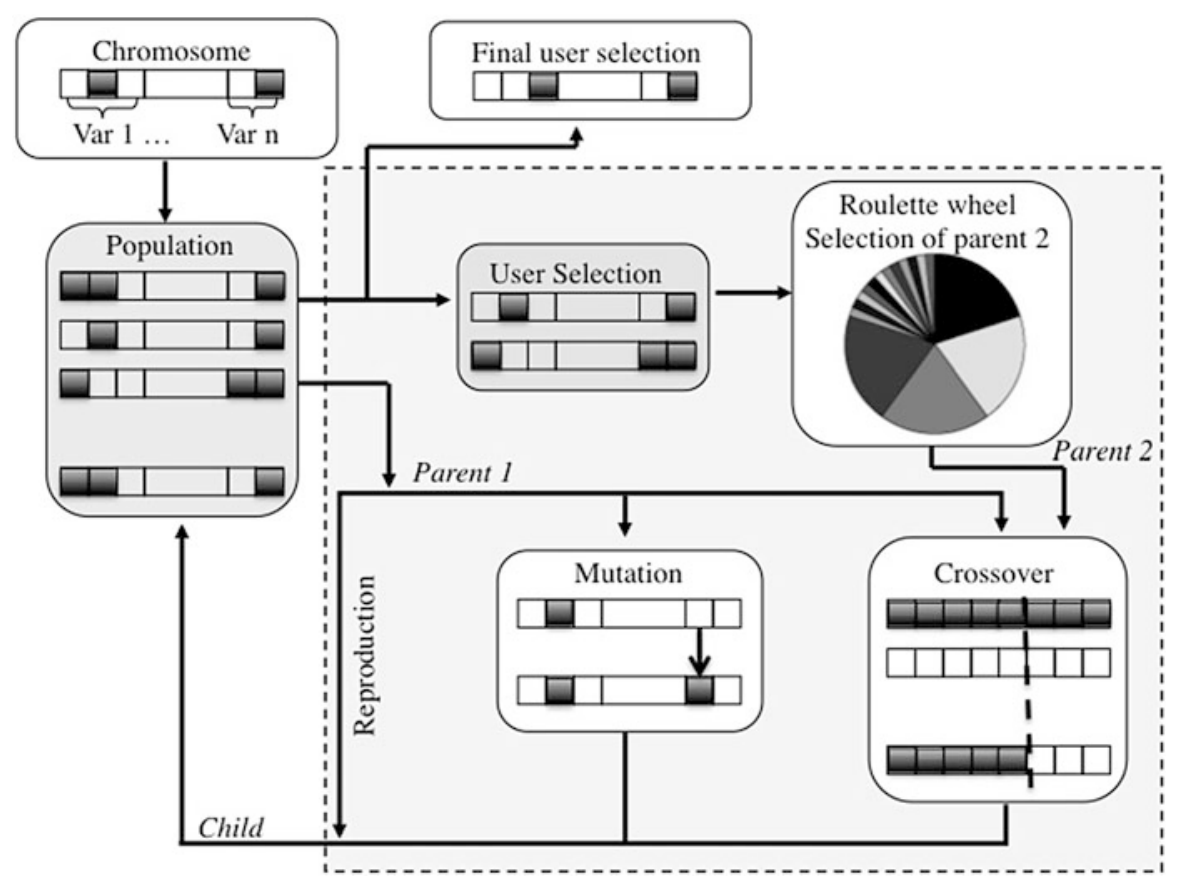

Fig. 3.1 Synoptic of the IGA process [14]

among the individuals of a given population, some solutions that seem better according to the constraints and objectives defined by the instruction of the test. This selected product are favored in the randomly election for the genetic operators. The most popular method of election is the roulette method, where individuals with better performances (selected by the user) have a higher probability of being selected (illustration in Fig. 3.1). The crossover operator consists in crossing several individuals with each other in order to generate better individuals. The mutation operator consists of modifying certain variables of a given individual in order to create a new, different individual and thus ensure that the search for solutions runs through the biggest design space as possible. Each generic operator has its own settings and the effectiveness of the generic algorithm strongly depends on these settings. Several studies are devoted to the influence of these parameters on the performance of the genetic algorithm [10, 12, 30].

By definition, in the MOGA-II algorithm [22], each chromosome of the population is a parent of the following generation, which gives robustness to the algorithm. A new population is thus created and evaluated, comparing each child to its parents. This iterative method runs until the program has reached the maximum of generations chosen by the experimenter or until the user estimates that the target has been reached. 
The efficiency of the IGA is ruled by its 3 operators: crossover, mutation, and selection, chosen randomly for each individual of the population. The rate at which these operations occur is determined by the value of their weights. The crossover rate $(R c)$, mutation rate $(R m)$ and selection rate $(R s)$ are real values chosen between 0 and 1 such that $R c+R m+R s=1$. An indicator, rand(i), is randomly chosen between 0 and 1 for each individual with a uniform distribution. (1) rand(i) $<$ Rc, the operation is a crossover. (2) $R c \leq \operatorname{rand}(\mathrm{i}) \leq R c+R m$, the operation is a mutation. (3) $\operatorname{rand}(\mathrm{i})>R c+R m$, the operation is a selection.

\subsubsection{Challenges of IGA}

The first difficulty in the use of IGA lies in the assessment of its fitness by the user: requiring the user to assess fitness can be time consuming or tiresome [26]. Gong [4] proposed to calculate the fitness by observing the time taken by the user to determine if an individual in a group is either "satisfactory" or "unsatisfactory". For example, products are presented to the user who is asked if it is "elegant". The users had to express their opinion on each product by clicking on "satisfied" or "not satisfied." This choice based method was compared to one where the users could rank their satisfaction on a sliding scale [6]. The task based on a slide required the evaluation of the entire population for each generation, which was tedious for the user. An individual's level of concentration influences their decision time, which can distort the results. Another way to assess the fitness is to use a choice-based scheme. In [24] the author's study a choice task for preference elicitation. The results showed that choices are practical to represent preference with a tolerable interaction effort and that algorithms based on binary choices are able to converge in a limited number of iterations.

Another difficulty in the use of IGA in user's tests lies in the convergence of the algorithm: an algorithm that is too slow may imply user's fatigued. To speed up the convergence, numbers of tools exist: krigging metamodel [16], chaining of neural network models and IGA [28] or cooperative interactive genetic algorithm based on the ratings of previous users [5]. If the acceleration of convergence is significant, it is limited to case studies with a reduced number of variables, because the choices can only be made on the products presented.

It is therefore, for our study, to find the right settings of the algorithm to have a reasonable convergence, that is to say, that give the user enough time to allow him to reach his target but not too much to tire him. To avoid the risk of premature convergence because of the selection operator, the mutation operator plays the role of "noise", jumping randomly in another part of the design space. Crossover will also explore new combinations. The difficulty is to balance their ratio. 


\subsubsection{Set up of the Genetic Algorithms}

To determine the most adapted crossover rate $(R c)$, mutation rate $(R m)$ and selection rate $(R s)$, we define an automatic mode. A virtual user simulates to evaluate products. It is assumed that the user has an idea in mind and the more he advances in the experience, the closer he gets to it. We therefore suppose coherence in his reasoning and his choices. The goal for the user is to ensure that the proposed products are getting closer and closer to the target he has in mind. To simulate the choices of a virtual user, a target product was arbitrarily chosen in the design space: $\mathrm{t}=\left[\mathrm{t}_{1}, \mathrm{t}_{2}, \ldots, \mathrm{T}_{\mathrm{n} \text { var }}\right]$. We calculate the distance to this target for each of the designs presented. The "selection" of the user is therefore logically composed of products that are the closest to the target. Calling this algorithm IGA is a misnomer since the algorithm is no longer interactive but for better understanding, automatic mode for IGA will be kept.

For each individual $j$, the distance $d(j, t)$ to the target was defined by the Euclidian distance function:

$$
d(j, t)=\sqrt{\sum_{i=1}^{n \text { var }}\left(\operatorname{rankx}_{i j}-\operatorname{rankt}_{i}\right)^{2}}
$$

with: rankt $t_{i}$ : rank of the level of the variable $t_{i}$ rankx $_{i j}$ : rank of the level of the variable $x_{i}$ for individual $j$

By assumption, the Euclidian distance function used was considered as representative of the perceptual distance of the user (tests with other distance functions showed that the IGA parameters were not too sensitive to the nature of the distance function used). The automatic mode was used to tune the parameters of the IGA [20]. This was done in order to be able to launch several simulations in the same conditions, and to have an average estimate of the convergence rates. Knowing the convergence rates allows designers to limit the design space (the number of variables and their levels) to a reasonable size so that the number of generations required for convergence can be decreased. It was estimated that a subject could process 30 generations in manual mode before becoming fatigued.

The objective is to assess, for a fixed number of generations, the average quality of the solutions provided by the IGA. The IGA is used in automatic mode (the fitness is simulated by the computation of the Euclidian distance to a given target). For the optimal set of IGA parameters, the IGA process was simulated $N$ times (Monte Carlo method). The quality of the solutions is assessed by two criteria: the average distance to the target of the best individual of the last generation $g$, and the average distance to the target of all the individuals of the last generation. The average distance to the target of the best individual of the last generation $d_{\text {best }}$ is given by: 


$$
\begin{gathered}
d_{\text {best }}=\frac{1}{N} \sum_{i=1}^{N} d^{g}\left(j_{\text {best }}^{i}, t\right) \\
j_{\text {best }}^{i}=\underset{j \in\{1, \ldots \text { popsize }\}}{\operatorname{argmin}}\left(d^{g}(j, t)\right)
\end{gathered}
$$

with: $j_{\text {best }}^{i}$ : the individual minimizing the distance to the target for simulation $i$

For each iteration of the IGA, the two individuals which minimize this distance are automatically selected. These individuals are considered as the closest individuals to the target. According to this selection, the IGA generates a new population of individuals.

We arbitrarily consider that the IGA converges and then stops the process if the distance between an individual, generated by the IGA, and the target is lower than 2 (difference of 2 ranks). The parameters in input of the Automatic IGA are (1) the number of variables, (2) the number of level for each variable (the same for all variables), (3) the "roulette" wheel rate (weight of a selected parent to create the next generation), (4) the mutation and crossover rates $\left(R_{m}\right.$ and $\left.R_{c}\right)$. Each one has coherent intervals of variation. For each set of IGA parameters, the IGA process is simulated $N=10$ times and the number of generations is measured. The mean value of this number of generation is computed, associated to the set of parameters. We estimated around 20 the number of generations which can be supported by a subject before fatigue. Consequently, we decided to select a size of problem defined by 5 variables and 5 levels for each variable for the rest of the study (the IGA, in automatic mode, needs 10 generations to converge for this size of problem). The best set of IGA parameters, for the size of problem, defined by 5 variables and 5 levels, with a Wheelrate of $16, \mathrm{R}_{\mathrm{m}}=0.15$ and $\mathrm{R}_{\mathrm{c}}=0.8$.

The results show a global improvement of the fitness of the population according to the $\mathrm{n}^{\circ}$ of generations: the average distance $d_{\text {average }}$ of a population to the target decreases with the number of generation and the quality of the population globally increases. The distance $d_{\text {average }}$ reaches a plateau above 60 generations (this distance is not null because the population is not homogeneous). The distance to the target of the best individual $\mathrm{d}_{\text {best }}$ also decreases, and is null above 60 generations. It signifies that above 60 generations, the method always converged toward the target for all the simulations $(N=100)$. For 30 generations, the distance $\mathrm{d}_{\text {best }}$ is approximately equal to 0.2. A study of the distribution of the distance $d^{g}\left(j_{\text {best }}^{i}, t\right)$ for all the simulations $(N=100)$ showed that $60 \%$ of the simulations converged perfectly toward the target, $30 \%$ converged toward the target with a difference of one level for one variable, and $10 \%$ converged toward the target with a difference of one level for two variables.

All these results make us confident in the ability of the IGA to converge when real subjects are used. 


\subsection{Application Case: Protocol and Results}

\subsubsection{Goal-Seeking Task}

To provide an example of the methodology, a study was conducted on a simple product common to every day French life; a wine glass. The digital mock-up of the glass (Fig. 3.2) was defined by 5 dimensional variables $\left(\mathrm{V}_{1}\right.$ to $\left.\mathrm{V}_{5}\right)$ with 5 levels.

Two tests were proposed to a panel of 20 subjects (age between 22 and 25), students at Ecole Centrale de Nantes: a goal seeking task and the research of the most "elegant" glass.

For both tests, the following conditions were set:

- The values of the IGA parameters: $R w=16, R c=0.8, R m=0.15$.

- The IGA were allowed to run through a maximum of 20 generations.

- If the subjects estimated that the task was fulfilled and the design selected satisfied, they could stop the test before the 20th generation.

- At the end of the entire process, the subject had to give the selected product a score from 0 to 10 , corresponding to the quality of the solution, with respect to the task.

The type of perceptive test was a choice of maximum $2(0,1$ or 2$)$ products on 8 presented. It was dimensioned because of the size of the screen ( 3 lines of 3 products), and the fact that the target stay visible during all the test thus, take the ninth place.
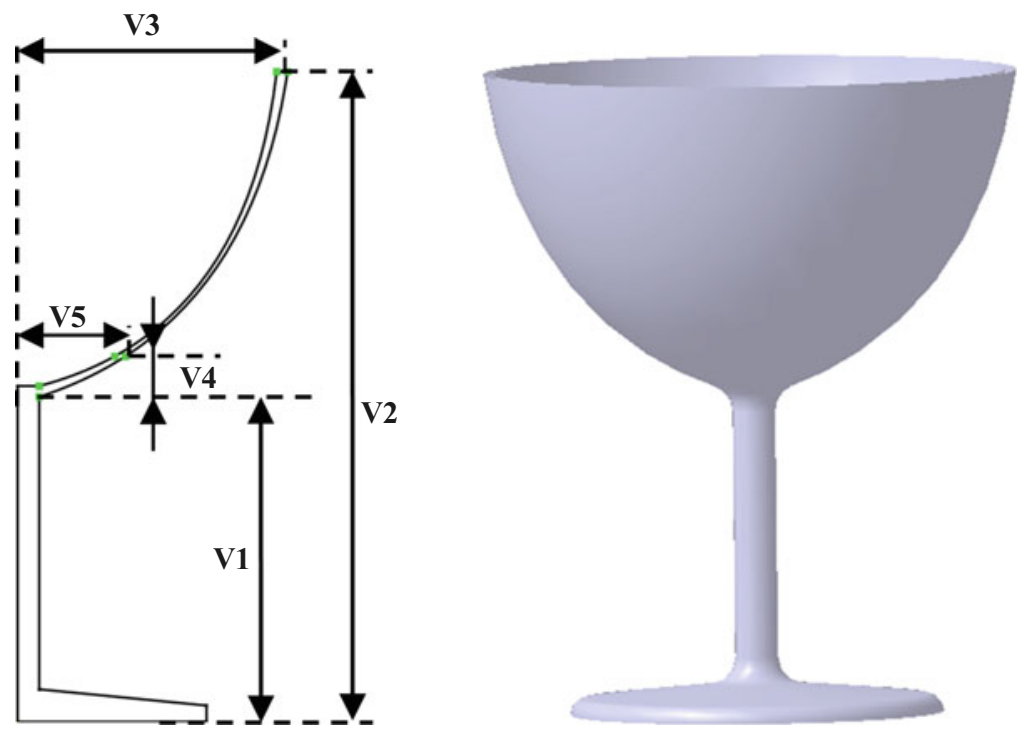

Fig. 3.2 Definition of the variables $\left(\mathrm{V}_{1}\right.$ to $\left.\mathrm{V}_{5}\right)$ to parameterize the geometry of the glass 
The test had three goals:

1. Warming up the users, familiarize them with the interface,

2. Confirming the performance of the method for the experimenter testing whether subjects were able to make the IGA populations converge toward a given goal,

3. Giving a feedback of the choice of the variables on this particular product.

Goal 1 and 2: Familiarization with the Task and Convergence of the Algorithm The test lasted an average of around $8 \mathrm{~min}$ per user. The target needed an average of 16.75 generations to appear. We estimated that it was a reasonable duration for a perceptive test. Around half of the subjects (Pop 1) stopped the algorithm before the last generation, meaning that they thought they had reached the target. This information confirms the hypothesis of our study.

\section{Goal 3}

To estimate the discrepancy between the target and the choices of the subjects, for each variable of the 5 variables, the standard deviation is calculated on the rank of the variable to avoid a scale effect. For example, V1 could take $i=5$ levels: $1_{1}=3.5$, $l_{2}=5, l_{3}=7,1_{4}=9,1_{5}=10$. The values themselves $l_{i}$ are not used but only the level $i$ are compared.

This standard deviation $\sigma_{\mathrm{j}}$ estimates, for each variable, the degree of agreement of the subjects' choices with the target (e.g. $\sigma_{j}=0$ if all the subjects chose the same level than the target for the variable $V j$ ). The results show that the 5 variables chosen do not have the same influence on the perceived shape of the glass. In Pop 1 , everybody chose a target with the exact level for V1 and V3. The conclusion was that the variations of these variables on the form were easy for the user to perceive. In contrast, the choices were more heterogeneous on V4 (the height of the point controlling the form of the glass). The differences were not perceptible in the same way for all variables, at least for the representation mode chosen. All the variables don't have the same perceptive influence on the form of the product. This result is useful for the designer to distinguish his product on more impacting parameters.

\subsubsection{Free Task on "Elegant” Glass: Protocol}

In this test, the subjects were not given a target glass, but were told to select the most elegant wine glass, according to their personal opinions. We assume that each subject has his/her own target in mind, and makes consistent choices during the test (no change of target). The objective function to minimize corresponds to the distance between the target in mind and the products presented. For example, if the ideal of elegance is a kind of champagne flute, the subject will be attracted by glasses with a long stem, small diameter and elongated, rejecting the glass of Fig. 3.2 for example.

Figure 3.3 describes the process of IGA, showing the exchange of data between the subject and the algorithm. A population of 8 glasses was firstly generated. We 


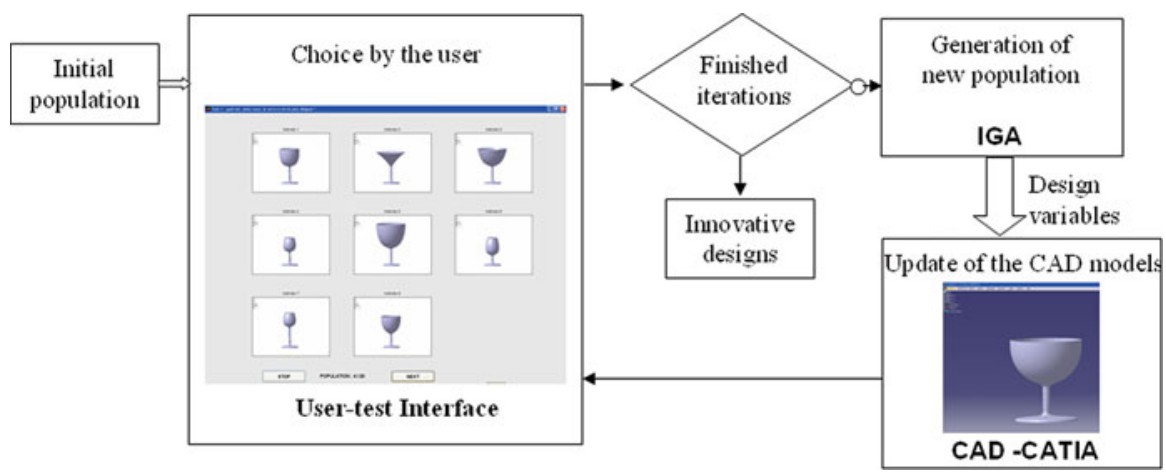

Fig. 3.3 Framework of the iterative user-test

keep 8 products, even if the 9th place dedicated to the target in the previous study is free, not to disturb the subject, to avoid being always considered as a target. This population is modeled in CAD tool (Catia V5 in our application) and presented to the subject via an interface (Matlab). Based on the choices done, a new population is generated and represented by new CAD modeling. The use of an Interactive Genetic Algorithm requires a constant exchange of data between the interface, the algorithm, the tool for product representation.

At the end of the process, the subject had to select one glass, and rate it on a scale from 0 (not at all elegant) to 10 (perfectly elegant). As in the previous test, the subject could stop the process and make their final choice before the 20th iteration (Fig. 3.3: "Finished iterations"? = yes).

The aims of the 2nd test were:

- To know to which extent the process converges toward a satisfying solution for the subject in the case of a simple product.

- To know if design trends concerning the elegance of a glass can be extracted from the results of the test. In other words, the problem was to uncover relevant information about the elegance concerning the design variables of the form.

- To estimate the variability of the results concerning the elegant semantic dimension, and to show how this information can be taken into account for product design.

\subsubsection{Free Task on "Elegant” Glass: Results}

Among the 20 subjects, 8 stopped the test before the 20th generation, giving a score of $10 / 10$ to the selected product. These 8 subjects were considered as perfectly satisfied with their choice. For the 12 other subjects, the average satisfaction score of their chosen glass was 8.3/10. This relatively high score and the low standard 


\section{Group3}

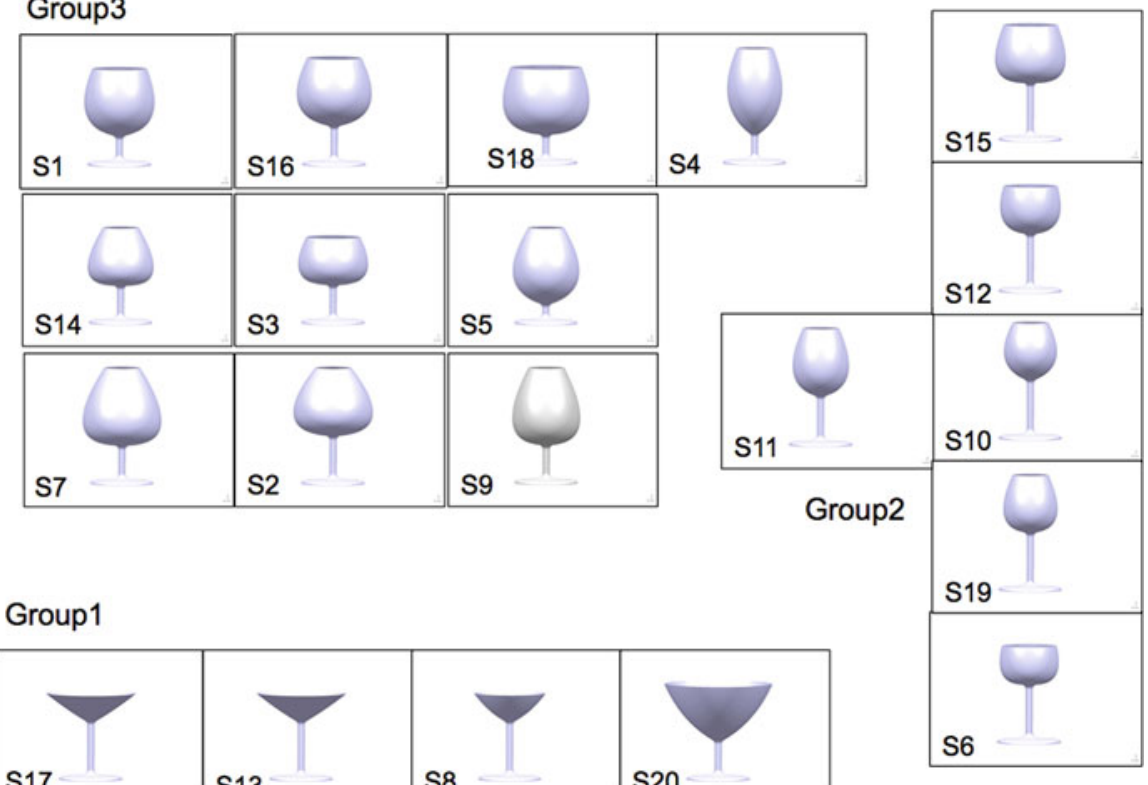

Fig. 3.4 Forms of the different glasses in the three groups

deviation imply that the subjects found satisfying enough glasses, and that 20 generations were sufficient to converge toward the desired product.

The first analyze considered the global population (20 subjects). Concerning the final glass chosen by the subjects, the results showed that only 2 subjects chose exactly the same glass. A great diversity in the final glass was noticed. The standard deviation of a subject compared to the 19 others was computed on each of the 5 levels. The results show that no variable was subjected to a great consensus concerning the elegance of the glass: the variability was of the same order for each variable, and no particular level could be considered as representative of the elegance. The conclusion led us to study more precisely the panel, searching for groups of subjects with similar image of elegance.

In order to provide a partition of the glasses and to define groups of targets similar from a perceptual point of view, a hierarchical ascendant classification (HAC) [7] has been performed. Details on this analyze can be found in [21]. To illustrate the potential of identifying homogeneous groups of subjects, three groups (HAC) were considered. The composition of the three groups is given in Fig. 3.4. While some of the users selected similar products, only S13 and S17 chose the exact same product.

To find a unique representation, a optimization was performed inside a group, aiming to minimize the dissatisfaction of the subject with the chosen representation. The results are presented Fig. 3.5.

The main result here is that the semantic dimension of elegance is subjective. There were at least 3 elegance types among only 20 students and on a total 


\begin{tabular}{|l|l|l|}
\hline Target 1 (Group 1) & Target 2 (Group 2) & Target 3 (Group 3) \\
\hline & \\
\hline
\end{tabular}

Fig. 3.5 Forms of the 3 proposed glasses, minimizing the dissatisfaction

population of $5^{5}$ glasses. A company could decide what type of elegance it wants for its glasses. That decision cannot be taken by the IGA methodology, as it is a strategic choice relating to the company's target values. One can also observe that the users have to be representative of the company's target user base. For example, Target 1 does not match a "traditional" wine glass form, which could signify that the users are not wine amateurs.

This test had to be considered as a pilot study, as the number of subjects was weak, and the product was relatively simple. This test allowed the description of the method and of the statistical data analysis, which could then used on a more substantial study with a more complex product.

\subsubsection{Conclusions on the Two Tests on the Glasses}

A goal-seeking test was carried out to give an estimation of the convergence abilities of the IGA, and revealed the problem of just noticeable differences in perception of forms. In Test 1, within a small number of generations, $80 \%$ of subjects either found a glass similar to the target, or one that they perceived to be the target (as they stopped the task before the end) in a less than 10 min. Perceptual tests with 20 subjects were conducted to demonstrate the validity of IGA and to conduct an analysis of the results. The perceptual tests on the semantic dimension - elegance of a glass were performed to show how the results can be analyzed and how to deal with inter-individual differences among users.

\subsection{Synthesis and Perspectives on the Use of IGA for Design}

The interest of IGA was previously demonstrated underlining the opportunities for design. If the case studied was simple, the methodology is adaptable to very different and more complicated product or system. The different steps must be broken down according to the study. 


\section{Preliminary step: product and representation.}

The first step is, the product/system defined, to choose which sensorial organ will be activate for the perceptive tests. In the example above, the visual sense was used but hearing can also be studied (concerning touch, smell and taste, the difficulty of generating new samples is for the moment an obstacle to the use of IGAs). The combination of both senses can be also imagined. Each representation has to be coherent with the goal of the designer. For example, a screenshot of a CAD model can be if needed completed in Virtual Reality Modeling Language (VRML), or a control of the sound level can be given to the subject. The interest of this method is that the representation of the product is independent of the algorithm (Fig. 3.3). The interface of this study is functional, and the computation time for updating the model is reasonable; in the wine glass test, the time between generations (time to built the $8 \mathrm{CAD}$-models) was less than $8 \mathrm{~s}$. The interface with the CAD software is a significant advantage. Replacing the glass by another product of 5 variables on 5 levels is immediate. To increase or decrease some of these values, a modification of the set up of the algorithm is simply needed.

Step 1: Variables and levels

Not totally independent of the previous step, the product must be configurable. The IGA can help the designer to reduce the complexity of his model. In the goal-seeking task, the subjects showed the convergence and divergence of results, meaning that their comprehension of the variables were different. We could therefore consider some parameters as not influential for the perceived quality of a product, which allows the designer to reduce his design space.

Step 2: parameterization of the algorithm and protocol

IGA implies the evaluation of the subject as fitness function. The human fatigue must be taken into account in the protocol, designing a tool limiting the duration and difficulty of the task. For the duration, the convergence must be studied, depending on the different parameters of the algorithm (mutation, crossover rates, wheelrate in the case of Moga II). The targeted task is an adapted tool for this step. Concerning the difficulty of the task, the choice made is a selection of 2 (maximum) on 8 products. If it seems affordable for a naïve subject, the quantity of information is not very important, especially increasing the number of variables, thus the design space. Other interventions of the user can be considered in the algorithm, not only on the evaluation of the fitness but also on the set up of the algorithm. For example, the subject could stop, even momentarily, the evolution of a criterion (variable) or reduce its scale and accelerate the convergence. He can also be asked the to score at the end of each step the product(s) he/she chose and one or two randomly chosen product from this generation. Hybrid versions of IGA, mixing evaluation of the fitness by or the machine or the human (not exclusively human), are also studied in this way. 
Step 3: analysis of results

A phenomenal amount of information is generated by such perceptive tasks. In the previous study, the distances between the selected product and the target or between the final population and the target is computed and studied. The IGA makes it possible to investigate the inter-individual differences for a group of users in the relationship between the semantics and the design features. It is possible to separate which attributes are common for the entire group, which attributes are specific to a subgroup of subjects, and which attributes are typical of a particular user. Other results can be exploited as the not chosen products. If a common characteristic appears in all the choices of the subject, he could reject some values of parameters, that is also very useful for the designer. The choice process could be redefine by asking the subject to select 3 categories of products: desired products, neutral products, rejected products. Modeling the user's path in the presented database can help to predict its next choices and thus accelerate convergence.

\subsection{Conclusion}

The general process for detecting design trends using Interactive Genetic Algorithms and user-tests was described. These tests are proposed to enhance innovation and to understand which design features are representative of a given semantic dimension. Applied to a simple example, it is adapted to other product and system (for example, layout problem [8]).

A limitation of the IGA algorithm used concerns the relative small size of the design space. To tackle bigger design spaces, several perspectives can be drawn. Concerning the algorithm, we could fit a model of the response of the subject (for example with a neural network) during the assessments, and optimize this response with classical GA. The tools of machine learning can be used to enrich the perceptive tests and modelize the behavior of the subject.

\section{References}

1. Aungst S, Barton R, Wilson D (2003) The virtual integrated design method. Qual Eng 15:565579

2. Desmet P (2003) Measuring emotion: development and application of an instrument to measure emotional responses to products, Human-computer interaction series, 3. Springer, Dordrecht

3. Goldberg DE (1989) Genetic algorithms in search, optimisation and machine learning. Addison Wesley, Reading

4. Gong DW, Pan FP (2003) Theory and applications of adaptive genetic algorithms. China University of Mining and Technology, Xuzhou

5. Gong D, Zhou Y, Li T (2005) Cooperative interactive genetic algorithm based on user's preference. Int J Inf Technol 11:1-10 
6. Gong DW, Guo GS (2007) Interactive genetic algorithms with interval fitness of evolutionary individuals, dynamics of continuous, discrete and impulsive systems, series B: complex systems and applications-modeling. Control Simul 14(s2):446-450

7. Hair JF, Tatham RL, Anderson RE, Black W (1998) Multivariate data analysis, 5th edn. Prentice Hall, Upper Saddle River

8. Hasda RK, Bhattacharjya RK, Bennis F (2017) Modified genetic algorithms for solving facility layout problems. Int J Interact Des Manuf (IJIDeM) 11(3):713-725

9. Holland JH (1992) Adaptation in natural and artificial systems: an introductory analysis with applications to biology, control and artificial intelligence. MIT Press, Cambridge, MA

10. Hong TP, Wang H-S, Lin W-Y, Lee W-Y (2002) Evolution of appropriate crossover and mutation operators in a genetic process. Appl Intell 16(1):7-17

11. Hopfer H, Heymann H (2014) Judging wine quality: do we need experts, consumers or trained panelists? Food Qual Prefer 36:1-2

12. Jilkova J, Raida $Z$ (2008) Influence of multiple crossover and mutation to the convergence of genetic optimization. MIKON 2008, XVII international conference on microwaves, radar and wireless communications in Poland

13. Kelly JC, Wakefield GH, Papalambros PY (2011) Evidence for using interactive genetic algorithms in shape preference assessment. Int J Prod Dev 13(2):168-184

14. Kelly J, Papalambros PY, Seifert CM (2008) Interactive genetic algorithms for use as creativity enhancement tools. In: Proceedings of the AAAI spring symposium, Stanford, CA, pp 34-39

15. Kim HS, Cho SB (2006) Application of interactive genetic algorithm to fashion design. Eng Des 38:224-237

16. Li M, Li G, Azarm S (2008) A kriging Metamodel assisted multi- objective genetic algorithm for design optimization. ASME J Mech Des 130(3):031401

17. Nagamachi M (1995) Kansei engineering: a new ergonomic consumer-oriented technology for product development. Int J Ind Ergon 15:3-11

18. Poirson E, Petiot J-F, Richard F (2010a) A method for perceptual evaluation of products by naive subjects: application to car engine sounds. Int J Ergon 40(5):504-516

19. Poirson E, Petiot J-F, Aliouat E, Boivin L, Blumenthal D (2010b) Interactive user tests to enhance innovation; application to car dasboard design. International conference on kansei engineering and emotion research KEER 2010

20. Poirson E, Petiot J-F, Aliouat E, Boivin L, Blumenthal D (2010c) Study of the convergence of Interactive Genetic Algorithm in iterative user's tests: application to car dashboard design. In: Proceedings of IDMME - virtual concept 2010 Bordeaux, France

21. Poirson E, Petiot JF, Boivin L, Blumenthal D (2013) Eliciting user perceptions using assessment tests based on an interactive genetic algorithm. J Mech De Am Soc Mech Eng 135(3): $1-16$

22. Poles S, Rigoni E, Robic T (2004) MOGA-II performance on noisy optimization problems. In: Proceedings of the International conference on bioinspired optimization methods and their applications, BIOMA2004, 11-12 October 2004, Ljubljana, Slovenia, pp 51-62

23. Qian L, Ben-Arieh D (2009) Joint pricing and platform configuration in product family design with genetic algorithm. In: Proceedings of IDETC/CIE 2009, San Diego, CA, USA

24. Ren Y, Papalambros PY (2011) A design preference elicitation query as an optimization process. J Mech Des 133(1):111004

25. Shabestari SS, Bender B (2017) Enhanced integrated sensitivity analysis in model based QFD method. In: Proceedings of the 21st international conference on engineering design (ICED 17), Vancouver, Canada, 4, pp 317-326

26. Swait J, Adamowicz W (2001) The influence of task complexity on consumer choice: a latent class model of decision strategy switching. J Consum Res 28:135-148

27. Takagi H (2001) Interactive evolutionary computation: fusion of the capabilities of EC optimization and human evaluation. Proc IEEE 89(9):1275-1296 
28. Tseng I, Cagan J,Kotovsky K (2011) Learning stylistic desires and generating preferred designs of consumers using neural networks and genetic algorithms. DETC2011-48642, ASME IDETC - design automation conference, Washington, DC

29. Yoshida S, Aoyama H (2008) Basic study on trend prediction for style design. ASME International Design engineering technical conferences, Brooklyn, New York, USA

30. Zhang J, Chung HSH, Zhong J (2005) Adaptive crossover and mutation in genetic algorithms based on clustering technique. In: Proceedings of the 7th annual conference on genetic and evolutionary computation, GECCO'05, Washington DC, USA - June 25-29. ACM, New York, pp 1577-1578. ISBN:1-59593-010-8, https://doi.org/10.1145/1068009.1068267 